\title{
Immature CML cells implement a BMP autocrine loop to escape TKI treatment
}

\author{
Mohammad Houshmand, Paola Circosta, Giuseppe Saglio \\ Department of Clinical and Biological Sciences, University of Turin, Turin, Italy \\ Correspondence to: Giuseppe Saglio. Department of Clinical and Biological Sciences, University of Turin, Turin, Italy. Email: giuseppe.saglio@unito.it. \\ Comment on: Grockowiak E, Laperrousaz B, Jeanpierre S, et al. Immature CML cells implement a BMP autocrine loop to escape TKI treatment. \\ Blood 2017;130:2860-71.
}

Submitted Apr 19, 2018. Accepted for publication Jun 11, 2018.

doi: $10.21037 /$ tcr.2018.06.10

View this article at: http://dx.doi.org/10.21037/tcr.2018.06.10

\section{Introduction}

Chronic myeloid leukemia (CML) is a clonal myeloproliferative disorder emanating from a reciprocal translocation between BCR on chromosome 22 and ABL kinase on chromosome 9, generally known as the Philadelphia chromosome. Although activity level of ABL kinase in the resting condition is preserved and regulated by a hydrophobic pocket, in CML, this pocket substitute with $\mathrm{BCR}$ that engenders in incessant activation of $\mathrm{ABL}$ and culminates in actuation of downstream signaling pathways $(1,2)$. Introduction of tyrosine kinase inhibitors (TKIs), revolutionized the response criteria, progression free survival and overall survival in CML patients in contrast with the previous form of treatment comprise of interferon- $\alpha$ and cytarabine combination (3). Even though a sizable proportion of cells respond to TKIs in a highly efficient manner, a strong intimation of resistance has been received from a minority group called leukemic stem cells (LSCs), responsible for disease progression (4). Impervious to TKIs whether by the acquisition of some features such as domain mutations or inheritance hallmarks, come to pass in a spatial structure called bone marrow niche. So, acts as a shield in the way of targeting LSCs and plays an indispensable role in the development of leukemia (5).

\section{Bone marrow niche}

Generation of leukemic niche refers to this fact that normal hematopoietic stem cells (HSCs) microenvironment become vanquished by the propensity of leukemic cells to evasion and persistence (6). In the leukemic condition, the interaction of CML stem cells with stromal cells mediated by CD44, selectins and their ligands and also mutual secretion of various soluble factors that propel CML cells into the quiescent state (7-10). It has been elucidated that expression of CXCR4 is downregulated in CML cells prior to treatment initiation. Instantly after applying tyrosine kinase inhibitors, CXCR4 is upregulated due to the direct correlation with BCR-ABL and cells migrate to the bone marrow milieu for the homing (11). This lodgment induces dormancy in CML cells and makes the bone marrow niche as a sanctuary for minimal residual disease (12). Various procedures have been taken into account in the bone marrow context to promote chemoresistance and one of the newly described one is attributed to bone morphogenic proteins (BMPs) family.

\section{Bone morphogenic proteins}

Bone morphogenic protein ligands as a member of transforming growth factor- $\beta$ superfamily (TGF- $\beta$ ), have a crucial role in the bone formation, evolving of gastrointestinal tracts, heart, and also the genesis of primitive hematopoiesis in the embryo. Activation of BMP signaling pathway occurred in a Smad dependent and independent pathway. In the Smad dependent manner, as the primary mechanism, following attachment of BMPs to constantly active type 2 receptor (BMPIIR), it phosphorylates type 1 receptor (BMPIR) and phosphorylation of the downstream Smads is conducted by BMPIR. Recruitment, formation and nucleus translocation of the transcriptional complex composed of Smad1, 5, 8 as R-Smads and Smad4 as co-Smad, regulates 
the gene expression process. These trends are governed and inhibited by Smad6 and 7 which suppress phosphorylation of the R-Smads (13). The contribution of BMP2, BMP4, BMP7 for the maintenance and self-renewal of HSCs is noteworthy, but perturbation of related signaling pathway in leukemia development is a formidable challenge (14). Align with these notions, a precious study about the role of BMP ligands has been carried out to shed light on the role of these double-edged proteins in induction of TKI resistance in primitive CML cells (15).

\section{BMPs, guardian against tyrosine kinase inhibitors?}

Grockowiak et al. measured the expression of BMPR1b transcript in 71 newly diagnosed CML patients. Elevated expression of BMPR $1 \mathrm{~b}$ in almost $40 \%$ of the cases referred to the importance of this element in CML pathogenesis. Following reaching complete cytogenetic response (CCyR), transcript level and also protein expression of BMPR1b in bone marrow CD34+ cells suppressed, but in contrary imatinib resistant population exerted a higher expression pattern. It became clear that imatinib has the ability to enhance the expression of BMPR $1 b$ in CD34+ CML cells invitro and indicated to new resistance mechanism in primitive cells. To find out whether this expression is concomitant with TKI resistance, LTC-IC and CFC assays was performed. Their results showed that cells expressing a higher level of BMPR1b were expanded and survived more in exposing to imatinib, INF- $\alpha$ and the combination than low expressing cells and this effect was intensified by the addition of BMP2 and BMP4 ligands. But how CML cells found the BMPs? Are there diverse resources for providing these proteins? Apart from mesenchymal stem cells (MSCs) in non-responder patients which secreted high amount of BMP4 in contrast with healthy one, a rise in transcript level of BMP2 and BMP4 in CD34+ CML cells of resistant patients was notable. However, patients follow up from diagnosis until reaching the CCyR or resistance state, declared a significant increase in BMP4 but not BMP2 in CML cells. This finding revealed that CML cells by production of BMP4 in an autocrine loop might tolerate TKI. Up-regulation of TWIST-1 as a modulator of tumor growth and drug resistance, was mediated by addition of BMP ligands. Also, inhibition of this transcription factor by BMPR1b inhibitor posited the role of BMP4-BMPR1b-TWIST1 axis in resistance adoption.

\section{Discussion}

A reasonable percentage of CCyR $(83 \%)$, event free survival $(81 \%)$ and overall survival $(85 \%)$ are achievable by considering imatinib as the first option for treatment of CML. But after a while emergence of resistance or tolerance attribute the liability to the bone marrow niche as the leading context where leukemic development is in progress $(16,17)$. Secretion of G-CSF and IL-3 and also BMPs in an autocrine manner admitted this point that CML cells depend on the state of the disease are self-supporting $(15,18)$. On the one hand, TWIST1 as a promoter of cell growth, survival and drug resistance has been reported to deregulated in CML patients. Imatinib suppresses the expression of this transcription factor but it remains high in the resistant clone which may refer to a decent prognostic factor for assessment of resistance in CML (19). On the other hand, adjustment of its expression by BMPs proved that an important target for breaking the wall of resistance came into existence (15). It has been elucidated that CML LSCs survive in a BCR-ABL independent fashion and in turn become contingent on Wnt- $\beta$ Catenin, PI3K-AKT, Hedgehog and other signaling pathways (20). Mutual interaction of BMPs with these pathways (21) may bring new insight for further investigation in targeting CML LSCs. Meanwhile, one study stated that activation of BMP signaling leads to adherence of CML cells to stromal cells and this contact is an ample opportunity for leukemic cells to evade TKIs (22). Another finding demonstrated that treatment of bone marrow mesenchymal stem cells with imatinib increases the osteogenic markers such as BMP2, osteocalcin, and Runx 2 and reflects the role of imatinib in niche modification (23). These concepts reiterated in acute promyelocyte leukemia which using ATRA downsized the expression of BMP expression in parallel with PML/ $\mathrm{RAR} \alpha$ fusion gene, however, in non-responder patients the expression pattern remained intact. They also posited the elevated expression of BMPs and related receptors in multiple myeloma and acute lymphoblastic leukemia reflect that BMPs family are a jack of all trades (24).

\section{Conclusions}

CML LSCs have shown to have an intrinsic resistance to TKIs, and they persist even in patients with CCyR, and this frequently results in the relapse after discontinuation of the TKI therapy. Part of this immunity germane to the formation of BCR/ABL independent mechanisms. So, 
ascertaining the compensatory pathways such as BMP would be an intriguing solution to eliminate CML LSCs.

\section{Acknowledgments}

Funding: Supported by AICR (Italian Association for Cancer Research) grant to G Saglio.

\section{Footnote}

Provenance and Peer Review: This article was commissioned and reviewed by the Section Editor Peipei Xu (Department of Hematology, The Affiliated Drum Tower Hospital of Nanjing University Medical School, Nanjing, China).

Conflicts of Interest: All authors have completed the ICMJE uniform disclosure form (available at http://dx.doi. org/10.21037/tcr.2018.06.10). The authors have no conflicts of interest to declare.

Ethical Statement: The authors are accountable for all aspects of the work in ensuring that questions related to the accuracy or integrity of any part of the work are appropriately investigated and resolved.

Open Access Statement: This is an Open Access article distributed in accordance with the Creative Commons Attribution-NonCommercial-NoDerivs 4.0 International License (CC BY-NC-ND 4.0), which permits the noncommercial replication and distribution of the article with the strict proviso that no changes or edits are made and the original work is properly cited (including links to both the formal publication through the relevant DOI and the license). See: https://creativecommons.org/licenses/by-nc-nd/4.0/.

\section{References}

1. Cilloni D, Saglio G. Molecular pathways: BCR-ABL. Clin Cancer Res 2012;18:930-7.

2. Hantschel O, Superti-Furga G. Regulation of the c-Abl and Bcr-Abl tyrosine kinases. Nat Rev Mol Cell Biol 2004;5:33-44.

3. Roy L, Guilhot J, Krahnke T, et al. Survival advantage from imatinib compared with the combination interferonalpha plus cytarabine in chronic-phase chronic myelogenous leukemia: historical comparison between two phase 3 trials. Blood 2006;108:1478-84.

4. Volpe G, Panuzzo C, Ulisciani S, et al. Imatinib resistance in CML. Cancer Lett 2009;274:1-9.

5. Crews LA, Jamieson CH. Selective elimination of leukemia stem cells: hitting a moving target. Cancer Lett 2013;338:15-22.

6. Houshmand M, Soleimani M, Atashi A, et al. Mimicking the Acute Myeloid Leukemia Niche for Molecular Study and Drug Screening. Tissue Eng Part C Methods 2017;23:72-85.

7. Krause DS, Lazarides K, von Andrian UH, et al. Requirement for CD44 in homing and engraftment of BCR-ABL-expressing leukemic stem cells. Nat Med 2006;12:1175-80.

8. Krause DS, Lazarides K, Lewis JB, et al. Selectins and their ligands are required for homing and engraftment of BCR-ABL1+ leukemic stem cells in the bone marrow niche. Blood 2014;123:1361-71.

9. Corrado C, Saieva L, Raimondo S, et al. Chronic myelogenous leukaemia exosomes modulate bone marrow microenvironment through activation of epidermal growth factor receptor. J Cell Mol Med 2016;20:1829-39.

10. Traer E, Javidi-Sharifi N, Agarwal A, et al. Ponatinib overcomes FGF2-mediated resistance in CML patients without kinase domain mutations. Blood 2014;123:1516-24.

11. Arrigoni E, Del Re M, Galimberti S, et al. Concise Review: Chronic Myeloid Leukemia: Stem Cell Niche and Response to Pharmacologic Treatment. Stem Cells Transl Med 2018;7:305-14.

12. Jin L, Tabe Y, Konoplev S, et al. CXCR4 up-regulation by imatinib induces chronic myelogenous leukemia (CML) cell migration to bone marrow stroma and promotes survival of quiescent CML cells. Mol Cancer Ther 2008;7:48-58.

13. Wang RN, Green J, Wang Z, et al. Bone Morphogenetic Protein (BMP) signaling in development and human diseases. Genes Dis 2014;1:87-105.

14. Zylbersztejn F, Flores-Violante M, Voeltzel T, et al. The BMP pathway: a unique tool to decode the origin and progression of leukemia. Exp Hematol 2018;61:36-44.

15. Grockowiak E, Laperrousaz B, Jeanpierre S, et al. Immature CML cells implement a BMP autocrine loop to escape TKI treatment. Blood 2017;130:2860-71.

16. Fava C, Rege-Cambrin G, Saglio G. Chronic myeloid leukemia: state of the art in 2012. Curr Oncol Rep 2012;14:379-86.

17. Bakker E, Qattan M, Mutti L, et al. The role of microenvironment and immunity in drug response in leukemia. Biochim Biophys Acta 2016;1863:414-26.

18. Jiang X, Lopez A, Holyoake T, et al. Autocrine production 
and action of IL-3 and granulocyte colony-stimulating factor in chronic myeloid leukemia. Proc Natl Acad Sci U S A 1999;96:12804-9.

19. Cosset E, Hamdan G, Jeanpierre S, et al. Deregulation of TWIST-1 in the CD34+ compartment represents a novel prognostic factor in chronic myeloid leukemia. Blood 2011;117:1673-6.

20. Holyoake TL, Vetrie D. The chronic myeloid leukemia stem cell: stemming the tide of persistence. Blood 2017;129:1595-606.

21. Guo X, Wang XF. Signaling cross-talk between TGFbeta/BMP and other pathways. Cell Res 2009;19:71-88.

Cite this article as: Houshmand M, Circosta P, Saglio G. Immature CML cells implement a BMP autocrine loop to escape TKI treatment. Transl Cancer Res 2018;7(Suppl 7):S722-S725. doi: 10.21037/tcr.2018.06.10
22. Kumar A, Bhattacharyya J, Jaganathan BG. Adhesion to stromal cells mediates imatinib resistance in chronic myeloid leukemia through ERK and BMP signaling pathways. Sci Rep 2017;7:9535.

23. Tibullo D, Giallongo C, La Cava P, et al. Effects of imatinib mesylate in osteoblastogenesis. Exp Hematol 2009;37:461-8.

24. Grcević D, Marusic A, Grahovac B, et al. Expression of bone morphogenetic proteins in acute promyelocytic leukemia before and after combined all trans-retinoic acid and cytotoxic treatment. Leuk Res 2003;27:731-8. 